\title{
SLEEP DISORDERS IN PATIENTS WITH MULTIPLE SCLEROSIS
}

\section{Authors:M. Chakroun ${ }^{1}$, S. Sakka ${ }^{1}$, O. Hdiji ${ }^{1}$, N. Farhat ${ }^{1}$, H. Hadj Kacem¹, M. Dammak ${ }^{1}$, C. Mhiri ${ }^{1}$. ${ }^{1}$ Habib Bourguiba university hospital, department of neurology, Sfax, Tunisia.}

\section{Introduction:}

Multiple sclerosis (MS) is associated with a diverse range of physical, cognitive, emotional, and behavioral symptoms. Sleep disturbance is a very common, yet underdiagnosed problem.

\section{Objectives:}

To assess the prevalence of poor sleep and investigate the factors that influence sleep quality of patients with MS.

\section{Methods:}

A cross-sectional and analytical study was carried out on 31 patients with MS, followed up in the neurology department of Habib Bourguiba hospital, Sfax, Tunisia, over a period of four months. Demographic data, duration of the disease and the disease course were extracted from patients' medical files. The quality of sleep was evaluated by the Pittsburgh Sleep Quality Index (PSQI) and the insomnia severity index (ISI) , anxiety and depression by the Hospital Anxiety and Depression Scale (HADS), the severity of fatigue symptoms by the Fatigue Severity Scale (FSS) and the level of disability by the Expanded Disability Status Scale (EDSS).

\section{Results:}

\section{Descriptive study:}

* The average age $=34,19 \pm 10$ years old. The average age at the onset of the disease $=25,06 \pm 5,7$ years

* Relapsing remitting MS occurred in $80,6 \%$ of cases.

* The mean EDSS score $=3,8 \pm 2,6$.

* The prevalence of insomnia was $61,3 \%$ and it was a severe insomnia in $22,6 \%$. with a delay of falling asleep in $25,5 \%$.

* The average number of hours of sleep was $6,8 \pm 3$ hours.

\section{Analytical study:}

\section{Insomnia was significantly correlated to :}

* depression $(p=0,001) /$ anxiety $(p=0,000)$ / fatigue $(p=0,01) /$ anti-depressant medication use $(p=0,026)$.

\section{Discussion:}

-Poor sleep is frequently observed in patients with MS, and previous studies have shown a prevalence ranging from 25 to $67 \%[1,2]$.

-Fatigue is one of the most frequent symptoms in MS [3,4]. The relationships between sleep disturbance and fatigue remain a matter of debate. Some researchers reported that no difference was found in the level of sleepiness between fatigued and non-fatigued patients with MS [3], while other authors found more significant relationships between sleepiness and fatigue [5-6].

-there is no specific guideline for treatment of insomnia in patients with MS. A therapy study revealed improvement in insomnia as a result of treatment of depression in MS [7], but many of the drugs involved have tolerance and addiction effects, which may limit their long-term use.

-Pain is often a comorbid condition to MS-related fatigue but is confounded by depression and medication for treating pain or, pain-induced sleep disorder. Depression is common in patients with MS. Our study showed that a significant correlation between depression and insomnia. Poor sleep can predict depression, and a depressed mood may be a predictor of a disrupted sleep [8].

-Cognitive Behavioral Therapy (CBT) is among the most effective treatments for depression, anxiety, insomnia, and a number of other mental health and behavioral conditions. CBT for Insomnia (CBT-I) is a type of CBT that specifically focuses on identifying and changing unhelpful thoughts and behaviors that interfere with sleep and it has been extensively studied and has been shown to be effective for reducing depression and improving symptom management in MS.

Conclusion: Poor sleep is more common in patients with MS than in the general population. Sleep disorders should routinely be evaluated in patients with MS to improve the quality of sleep among them. References : [1] Tachibana N, Howard R, Hirsch N, Miller D, Moseley I, Fish D (1994) Sleep problems in multiple sclerosis. Eur Neuro 34:320-323 [2]

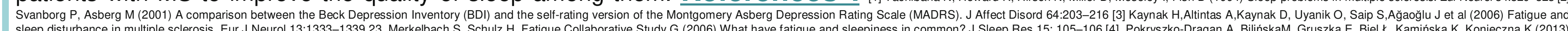

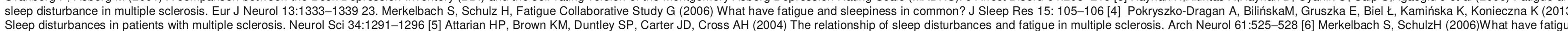

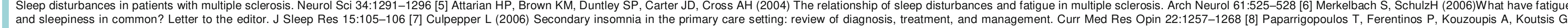

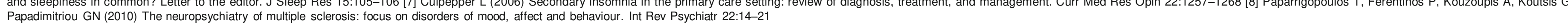

